\title{
Using Evidence-Based Practice: A Case Study
}

\author{
Carol Dickinson and Jayne Jackson
}

\section{Practice Paper}

Keywords: behaviour management, collaborative problem-solving model (CPS), evidence-based practice (EBP)

\begin{abstract}
This research reports on the application of aspects of a model of evidence-based practice (EBP) which were used to review a behaviour management programme. Special education practitioners employed at the Aotearoa/New Zealand Ministry of Education (MOE) drew from MOE principles and practices, as well as their professional expertise as they reviewed and critiqued the Collaborative Problem Solving Model (CPS). This study reveals two critical aspects when applying an evidence-based practice (EBP) process: understanding of research, and using a structured process. The article discusses a range of benefits and reports on the compatibility of the behaviour management programme with MOE principles and practices.
\end{abstract}

\section{INTRODUCTION}

Students in the Waikato area who exhibit persistent and difficult behavioural challenges are referred to the Hamilton Ministry of Education (MOE) Severe Behaviour Service. These children require intensive support in and out of school from the early intervention and school severe behaviour teams which is delivered within the education and learning setting. The MOE regularly considers evidence of effective programmes from the research literature as a means of strengthening its services in this area. This article reports on how a model of EBP was used as a tool to critique a potential new approach.

\section{EVIDENCE-BASED PRACTICE}

EBP was initially developed to inform medical practitioners (Biesta, 2007). Evidence-based medicine refers to "the integration of the best research evidence with clinical expertise and patient values" (Sackett, Rosenberg, Gray, Haynes \& Richardson, 1996, p. x).
EBP now extends to other contexts such as education, psychology, dentistry and nursing (Trinder \& Reynolds, 2000), and has been recommended and adopted in fields such as social work, probation, human resource management and education (Sackett, et al., 1996).

Since its inception in the 19th century, EBP has been a widely debated topic. The driving force behind the promotion of EBP is societal expectations on professionals to provide best current evidence (Department of Health, 1998) and best practice in providing quality services (Morris \& Mather, 2008). Current views of EBP identify 'best' evidence as deriving from a combination of research, practitioner knowledge and client knowledge. In a New Zealand educational context, action is dictated by EBP that combines these three core elements (Shlonsky \& Gibbs 2004). In addition, elements such as the 'expert' (medical) model, trial and error, a process for mediating information, and sharing experiences and identifying patterns have also been recognised as contributing to EBP by the Ministry of Education (2012). Figure 1 illustrates this integration in a Venn diagram developed by Bourke, Holden and Curzon (2005). EBP is located at the intersection of research, practitioner knowledge and clients' knowledge in an attempt to respect all parties' views as valid and important.

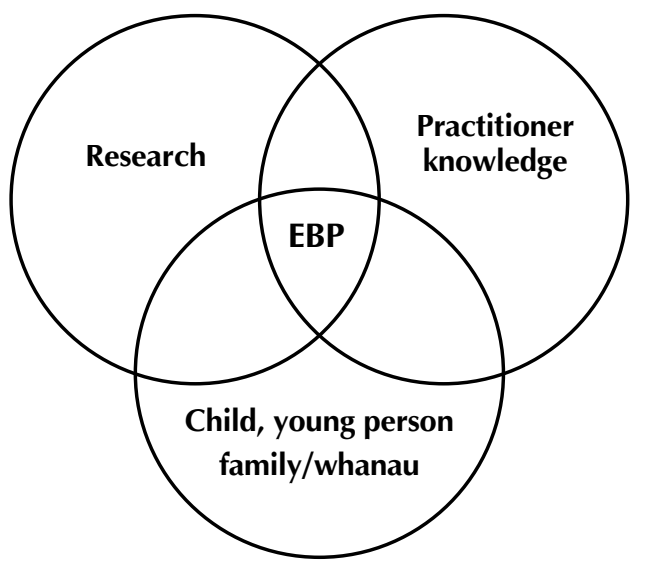

Figure 1. The collective elements of Evidence Based Practice

Bourke, Holden \& Curzon (2005) 
It is through mediation between research, practitioner and client that shared understanding of perspectives and desired outcomes, and negotiation of the process of collecting evidence is established (Ministry of Education, 2012). In Aotearoa/New Zealand, EBP further includes the cultural connection with Maori through their epistemology and genealogy (Ministry of Education, 2008) and "honoring the Treaty of Waitangi, which is the principle founding document of this land" (Paenga, personal communication, 7 April, 2013). This point of view is congruent with commentators on the Treaty including Durie who stated "The Treaty of Waitangi was seen as a unifying framework which accommodated partnership and power sharing" (2003 p. 101). Additionally, Parata (2009) asserted "The Treaty of Waitangi is a valued relationship management tool, symbolic of our past and central to our future" (cited in Macfarlane, 2009, p. 44).

In conventional use, evidence is defined as "information that is helpful in making the right decision" (Graham, 2003, p. 9). However, in professional settings, evidence needs to be of a sufficient quality to support a claim. Sackett et al., (2000) proposed that the word 'evidence' should be limited to information obtained from systematic clinical trials and if these were unattainable, the next - best, external evidence available. However, what counts as evidence is debatable (Maxwell et al., 2004; Morrison, 2001). Bateman (2006) states that the term 'evidence' will hold different meanings for different people as diversity in culture, worldview and experiences all influence individuals and specific groups' interpretation and justification of the term.

In the New Zealand education sector, questions have been raised as to what constitutes as 'evidence' and who is involved in creating a clear definition of the concept (Bateman, 2006). Uncertainty surrounding a clear definition of the term 'evidence' suggests risks in the application of international EBP in New Zealand, particularly in relation to the appropriateness of assessment and planning for New Zealand's indigenous (Māori) children and their whanau (Bateman, 2006). Despite these dilemmas, research demonstrates that across a variety of settings and populations a shared expectation exists that the fundamental goal of EBP is to provide 'best' current evidence, and 'best' current practice in contributing quality services.

\section{METHODOLOGY}

The current article was drawn from a larger study where aspects of a model of Evidence Based Practice were applied to evaluate a behaviour management tool called Collaborative Problem Solving (Greene,
2006). The Collaborative Problem Solving (CPS) model was originally developed in a clinical setting in the United States by Greene (2008). CPS has been described as an "evidence-based cognitive, behavioural, psychosocial treatment approach including a combination of developmental theory, systems theory and social learning theory" (Greene, 2010, p. 193) and neuroscience research. The aim of the CPS model is for adults and children to identify underdeveloped cognitive skills, and through collaborative problem solving, learn to solve the problems precipitating challenging behaviour, while simultaneously learning new skills.

A team of six special education practitioners employed by the Ministry of Education working in a severe behaviour team engaged in an evidencebased review of the CPS model. Participants attended a series of three focus group sessions where they used their knowledge of MOE principles, effective practices, and their individual professional expertise to evaluate the CPS model.

Reading material on the CPS model was provided to the participants prior to the first focus group session so that they could become familiar with the approach. Material included Greene's books "Lost at School," (2009), "The Explosive Child" (2010), and Greene and Ablon's (2006) book "Treating Explosive Kids: The Collaborative Problem Solving Approach". Additional resources included Greene's (2014) website "Lives in the Balance" an introductory powerpoint on CPS developed by the researcher, reviews and articles of the CPS literature (Diller, 2001; Greene, 2010 - Rennicke, 2008), and copies of published research on CPS (Pollastri, Epstein, Heath \& Ablon, 2013).

Session One ensured that all participants had an understanding of the CPS model. In order for participants to engage in an evidence-based evaluation of CPS, they would first have to have a thorough understanding of the programme. Following discussion of the CPS material, the participants had further questions so they sent an email to Greene for clarification.

Session Two began with a review of session one: the groups understanding of CPS. Greene's reply to the groups email was shared and discussed. Having established an understanding of CPS, the group could then begin to use their practitioner knowledge to critique the programme. The participants provided consensus statements of the model's strengths and cultural relevance in relation to the Ministry of Education practice guidelines and local context, and created a partial list of concerns with the CPS model. 
Session Three began with a review of the CPS model's previously identified strengths, shortcomings, and cultural appropriateness in the local context from the preceding session. Participants continued to evaluate the CPS model using their practitioner knowledge. Consensus statements were made regarding the model's potential value to contribute to effective and culturally-responsive education psychology practice in Aotearoa/New Zealand. The group provided a summary and recommendations of the CPS model should aspects of the programme be adopted for Ministry practices.

\section{DATA COLLECTION}

Data collected during the focus group discussions consisted of audio recordings, meeting minutes, participant notes, and discussion notes taken by the moderator, artefacts emerging from the discussion and a visual model of the CPS approach. Participants authenticated the data at each meeting. Data was used to explore the varied sources of knowledge, views, experiences and perceptions of the participants.

\section{FINDINGS AND DISCUSSION}

This study provided an opportunity for a group of six practitioners working at the Aotearoa/New Zealand Ministry of Education to engage in an evidence-based review within the Waikato context. The evaluative process used in this study was consistent with the Ministry's practice principle for an EBP approach where professional knowledge is shared. Goss and Leinback (1996) state "the opportunity to be involved in decision making processes, to be valued as experts, and to be given the chance to work collaboratively with researchers can be empowering for many participants" (cited in Sagoe, 2012, p. 5).

During all three focus-group sessions, diverse views were shared by participants. This led to rich discussions regarding queries, concerns, strengths, professional knowledge and experiences, and suggested modifications to the CPS programme. For authentic and sometimes controversial topics to be discussed, the focus-group had to have a supportive environment. In the current study, the focus-group provided an environment that was conducive to engaging in sharing, learning and ultimately contributing to each person's knowledge base.

A number of themes emerged from the data. The discussion below explores collegiality and the collective voice, culturally-specific experiences and perspectives, communication with Greene, and elements of CPS identified as congruent and incongruent with MOE principles and practices.

\section{Collective Voice and Collegiality}

All the participants agreed that a strength of the study was the opportunity to have an individual as well as a collective voice in the decision-making process. Speaker Three commented: "I have enjoyed the opportunity to share my experiences and knowledge of work in this field with my colleagues as it is satisfying knowing that I have made a valuable contribution to Ministry practice". Speaker Four went on to say: "This experience has brought the team closer together. There is a strong sense of respect and pride in knowing I work with a competent group of professionals with whom I share commonalities as well as differences. This opportunity enabled me to connect on a level I had not experienced with my colleagues prior to the study. Although I know the collective voice is a powerful tool for sharing and exploring new ideas and for gaining greater insight into skills, knowledge and experiences of my colleagues, what I got out of it was also a personal achievement. This reciprocated process provided the foundation for greater understanding and made the process enjoyable".

From these statements it is clear that these participants found the process of collaborative evaluation useful as a tool for their own professional development as it provided opportunities to learn from, and with, their colleagues. Through validation and support of this knowledge, the participants can be encouraged to further develop effective practices. Speaker One shared: "I've learned so much from my colleagues through this process. Through listening to their personal experiences, I learned what our service looks like through different lenses and in many instances this was quite different to mine".

\section{Culturally-specific Experiences and Perspectives}

The varied cultural experiences and perspectives of the participants in the group added an element of strength to this study in that the participants were able to contribute new information to research, and learn from each other in the process. This study was situated at the Ministry of Education Waikato office so was culturally-specific to the participants and to the Waikato district. The participants in the study represented different cultural groups which provided varied ethnic experiences. Cultures represented were New Zealand Māori, South African, British and New Zealand European. Some of the participants shared that they had learned something about their colleagues' culture that they did not know before they had started the study. For example, Speaker Two shared: "I didn't realise that the types of questions I asked Māori students limited the amount of information I was able to access". Speaker Five went 
on to say: "I work with my colleagues every day so thought I knew them really well until I took part in this study. I learned so much more about them and their approach to working with children and families. I feel humbled by the experience". Speaker One concluded: "I realised through this experience that there is more to culture than someone's language, customs, values and beliefs. I learned that it is the unspoken culture (relationships and sense of belonging within a culturally-diverse group of people) that connects people and this is what I experienced from this study". The effect of greater understanding or māramatanga "enables a person to develop new beliefs about one's self plus their ability to effect change within the self and within their relationship with others" (Te Raki Pae Whanau Support and Counselling Centre, p.1, 1999). Durie (2001) states "Relationships are a source of learning, empowerment, and identity for all of us. This is reflected in the concept of whanaungatanga" (p. 200). Whanaungatanga is about, "taking the time to listen and respond, rather than persuade and coerce others to see things in the same way as we do" (Durie, 2001, p. 200).

\section{Communication with Greene}

A further strength of this study was the direct communication the participants shared with Greene. The strength of interacting with Greene as the originator of CPS ensured that firstly the participants had a thorough understanding of the model. Secondly, there was rigor in applying the EBP model because the participants had interacted sufficiently with the research material. Mishler (2002) states "Measures of reliability and validity are replaced by the ideas of trustworthiness, which is "defensible" (p. 282) and "establishing confidence in the findings" (Lincoln \& Guba, 1985, in Golafshani, 2003, p. 602). Speaker Two expressed "By directly contacting Greene, it reduced the erroneous perception we could have taken from his studies and his books". Speaker Four added "It was great to get some clarity around some of the questions we put to Greene and I appreciated his feedback on some of our views. He didn't just answer the questions, he responded to what we had to say as well and this enabled me to make a connection with him albeit from a distance". Speaker One concluded "Once I had made this connection with Greene, I found myself more interested in delving further into the CPS material. My view of the CPS model then changed from one of instant critique to viewing it from a position of greater understanding".

\section{Congruent and incongruent aspects}

Discussion concluded that some elements of CPS were congruent with MOE principles and practices, and other aspects were incongruent. Congruent aspects included: theory of behaviour; collaborative and coordinated assessment; delivery of the service; inclusive practice; building relationships; integrity, and diagnosis. Incongruent aspects included: the use of the term 'collaborative'; criteria for Ministry behaviour service; Greene's philosophy; theory of behaviour; the referral and informed consent process; assessment practice; cultural relevance; relationship-building and the prescriptive nature of the intervention. Although these findings enabled the participants to make informed decisions about the usefulness of CPS, the focus of this article is on the process of EBP that the participants undertook.

In concluding the evidence-based review, the participants agreed that as the CPS model stands, they would not adopt it as other models of practice better-fit an Aotearoa/New Zealand context. The participants also concluded that any adaptations to the CPS model would alter its effectiveness as an EBP.

\section{Child / Young Person and Family EBP Practice Contributions}

Based on the EBP Venn diagram (see Figure 1) illustrated in the literature review, this research sits in the overlap between practitioner knowledge and research. However, EBP is considered to be derived from the integration of research, practitioner knowledge, and the life experiences of the family, whanau, child and young person. Although only the two elements of EBP, practitioner knowledge and research, are represented in this study, the findings are grounded in the field. A complete evidence-based evaluation would need to include parents, children and teacher's perspectives which were beyond the scope of this study.

\section{CONCLUSION}

Within this framework of practice, literature recognises how different fields of professional expertise develop their own criteria and standards to evaluate 'quality' evidence based on a contextual frame of expertise. EBP is determined in different ways by different people and that criteria reflect the unique qualities of each field that uses it. In the current study there were two critical aspects when applying the evidence-based model; understanding the research, and engaging in a structured review. Furthermore, important benefits were evident for the participants and the researcher. The two critical aspects used to apply the evidencebased model in the current study reflect the criteria the participants used to evaluate the CPS model within the Waikato context. 


\section{Understanding the research}

A critical element to the success of any evidencebased evaluation is developing a deep understanding of the research. In the current study, the process of active engagement with the research materials occurred through providing participants with articles, reviews and readings on the CPS model, direct communication with Greene, and rich discussion of the research evidence. Without a rigorous understanding of the material the process of evidence-based review is compromised.

\section{Structured process}

When using practitioner knowledge to critique a programme, a structured process was useful. In the current study, the participants considered which aspects of the CPS were congruent and incongruent with Ministry principles and practices and also with their own professional experience. This process enabled the participants to engage in purposeful discussion and make valid conclusions.

\section{Benefits}

The professional benefits of engaging in an EBP review went beyond an evaluation of a specific programme. Participants also reported on the importance of having their voice heard, which led to enhanced professional confidence. The value of developing a 'safe' environment is that people can freely and naturally communicate their views and ideas, within a culture of respectful listening. Additionally, participants reported the value of being able to learn from their colleagues. Both of these led to enriched practice.

Although this research looked at two aspects of EBP, research and practitioner knowledge, the authors of this article would recommend a further phase in the application of an EBP: consultation with child/ young person, family/whānau as the client's opinions, experiences, knowledge and values need to be considered.

\section{REFERENCES}

Bateman, S. (2006). Evidence based practice in special education: Implications for indigenous (Māori) consumers. Retrieved from http:// review.mai.ac.nz/index.php/MR/article/ viewPDFInterstitial/21/21

Biesta, G. (2007). Why "what works" won't work: Evidence based practice and the democratic deficit in educational research. Educational Theory 57(1), 1-22.

Bourke, R., Holden, B., \& Curzon, J. (2005). Using evidence to challenge practice. A discussion paper. Wellington, NZ: Ministry of Education.

Department of Health (1998). A first class service: Quality in the NHS. London, UK: HMSO.

Diller, L. H. (2001). Defusing the explosive child and the demise of discipline. Retrieved from http:// www.salon.com/2001/07/18/discipline/singleton/

Durie, M. (2001). Cultural competence and medical practice in New Zealand. Paper presented at Australian and New Zealand Boards and Council Conference, 22 November. Wellington, NZ.

Durie, M. (2003). Whaiora: Māori health development. New York: Oxford University Press.

Golafshani, N. (2003). Understanding reliability and validity in qualitative research. The Qualitative Report 8(4), 597-607.

Goss, J. D., \& Leinback, T. R. (1996). Focus groups as alternative research practice, Area 28(2), 136-142.

Graham, P. (2003). What is evidence? ACPP Occasional Papers 22, Mental Health.

Greene, R.W. (2006) Colloborative Problem Solving

Greene, R. W. (2009). Lost at School: Why our kids with behavioural challenges are falling through the cracks and how we can help them. NY, USA: Scribner.

Greene, R. W. (2010). The explosive child. A New Approach for Understanding and Parenting Easily Frustrated, Chronically Inflexible Children. NY, USA: Harper Publishing.

Greene, R.W. (2014). Lives in the balance. Retrieved from: http://www.livesinthebalance.org/

Greene, R.W., \& Ablon, J.S. (2006). Treating explosive kids: The collaborative problem-solving approach. NY: USA: Guilford Publications.

Macfarlane, S. (2009). Te pikinga ki runga: Raising possibilities. Set (2), 42. Retrieved from http:// www.nzcer.org.nz/nzcerpress/set/articles/tepikinga-ki-runga-raising-possibilities 
Maxwell, G., Robertson, J., Kingi, V., Morris, A., \& Cunningham, C. (2004). Achieving effective outcomes in youth justice: An overview of findings. Wellington, NZ: Ministry of Social Development.

Ministry of Education (2008). Ngā haeata mātauranga 2006/07: Annual report on Māori education. Wellington, NZ: Ministry of Education.

Ministry of Education (2012). Springboards to practice. Retrieved from http:/www.minedu.govt.nz/ NZEducation/EducationPolicies/SpecialEducation/ PublicationsAndResources/ResourcesForEducators/ SpringboardsToPractice.aspx

Morris, R. J., \& Mather, N. (2008). Evidence-based interventions for students with learning and behavioral challenges. New York: Routledge.

Morrison, K. (2001). Randomised controlled trials for evidence-based education: Some problems in judging 'what works.' Evaluation and Research in Education, 15, 69-83.

Paenga, T. (2013). (personal communication, March, 2013)

Pollastri, A. R., Epstein, L. D., Heath, G. H., \& Ablon, J. S. (2013). The collaborative problem solving approach: Outcomes across settings. Collaborative Problem Solving 21(4), 198.

Rennicke, C. (2008). Cognitive behavioural therapy book review 4(5), 1-3. Retrieved from http://www. Cognitive TherapyAssociation.org/cbtbr.aspx

Sackett, D., Rosenberg, W. M., Gray, J. A., Haynes, R. B., \& Richardson, W. S. (1996). Evidence based medicine: What it is and what it isn't. Retrieved from http://www.bmj.com/cgi/content/ full/312/7023/71

Sagoe, D. (2012). Precincts and prospects in the use of focus groups in social and behavioural science research. The Qualitative Report, 17(29), 1-16.

Shlonsky, A., \& Gibbs, L. (2004). Will the real evidence-based practice stand up? Teaching the process of evidence-based practice to the helping professions. Brief Treatment and Crisis Intervention, 4(2), 137-153.

Te Raki Pae Whanau Support and Counselling Centre (1999, July). The dynamics of Whanaungatanga - Te ao o te tangata. An abstract of the workshop presented at Children and Family Interventions Conference, Wellington, New Zealand.

Trinder, L., \& Reynolds, S. (2000). Evidence based practice: A critical appraisal. London: Blackwell Science Limited.

\section{AUTHOR PROFILES}

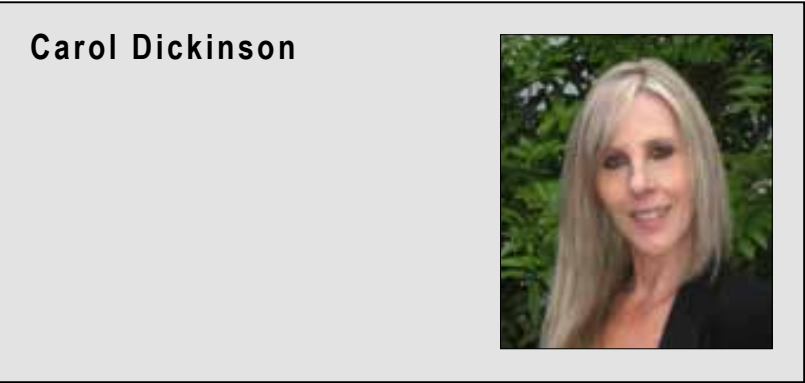

Carol Dickinson I am currently employed by the Ministry of Education in Hamilton. I have worked in the area of Special Education for the past five years and prior to that I was a teacher for 12 years, five of which were in special schools in the UK. I have owned my own business 'Obehave' where I was contracted by schools, parents and CYFS to work with children with challenging behaviour. I have completed Post Graduate Diplomas in Education (Guidance Studies, and Psychology) and a Masters in Educational Psychology at Massey University, Albany, Auckland. I am currently completing an internship Educational Psychology. Appreciation is expressed to Ministry of Education Hamilton for providing the setting that made this data collection possible, and to the colleagues who participated.

I live in Hamilton with my partner Jason. My two daughters Kylie and Nicky live in Napier along with my three grandsons Jai (5), Van (4) and Dylan (1). During my leisure time I work out at the gym and relax with family.

\section{Email:}

carol.dickinson@minedu.govt.nz

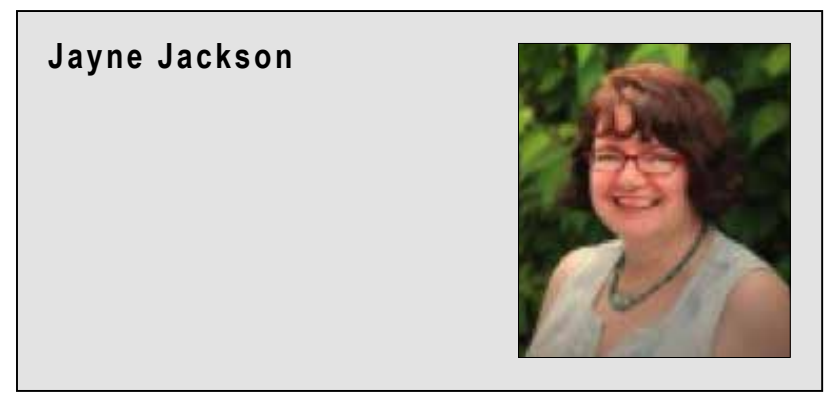

Jayne Jackson I am a lecturer in the Institute of Education at Massey University on the Albany Campus where most of my work is in the area of pre-service teacher education. I spent 17 years as a school leader and classroom teacher in Auckland and the USA before moving into tertiary education. I'm passionate about all children having positive experiences at school. I am currently completing a Doctorate through Massey University. I live in Albany with my husband Graham. When I'm not at work, one of my favourite places to be is on our boat in the Hauraki Gulf.

\section{Email:}

J.H.Jackson@minedu.govt.nz 